\title{
Molecular Interplay at the Membrane and Impact on Cellular Physiology
}

\author{
Thomas Pucadyil $^{1} \cdot$ Kiran Kulkarni $^{2} \cdot$ Durba Sengupta $^{2}$
}

Accepted: 4 May 2021 / Published online: 26 May 2021

(c) The Author(s), under exclusive licence to Springer Science+Business Media, LLC, part of Springer Nature 2021

The cell surface is the playground for rich biological phenomena. These emerge from short-range interactions resulting in the nanoscale organization of membrane proteins and lipids, the conformational dynamics of membrane proteins and their interactions with lipids as well as topological changes in membrane form and structure. Such phenomena cooperate to manifest into important cellular phenomena such as cell adhesion, signal transduction, secretory and endocytic trafficking, and cytoskeletal dynamics. In fact, differences in membrane composition and organization can lead to diseased conditions, even cell death. Lipid membranes are also considered as early signatures of life as they demarcate living matter into cells. Consequently, an understanding of membrane processes requires an appreciation of the membrane from a 'living matter' perspective that signals within, into, and out of the cell.

Last year (when we still could) we organized a 4-day long International Symposium on Cell Surface Macromolecules (ISCSM 2020) held at the Indian Institute of Science Education and Research (IISER) and the National Chemical Laboratories (NCL), both located in Pune, India. The aim of the meeting was to address this molecular interplay by gathering a multidisciplinary cast of speakers with expertise in physics, molecular modeling, and biology. The ISCSM is part of a series held once in 3 years and has been instrumental in establishing the core of interdisciplinary scientists working on membrane biology. The meeting gathered 112 attendees from far and wide, with 41 speakers of which seven

Thomas Pucadyil

pucadyil@iiserpune.ac.in

$\triangle$ Kiran Kulkarni

ka.kulkarni@ncl.res.in

$\triangle$ Durba Sengupta

d.sengupta@ncl.res.in

1 IISER Pune, Dr. Homi Bhabha Road, Pune 411 008, India

2 CSIR-National Chemical Laboratory, Dr. Homi Bhabha Road, Pune 411 008, India were early career scientists. The meeting showcased work from 16 students and postdocs and offered up the opportunity for several attendees to take home coveted poster prizes sponsored by scientific journals and societies. Of the forty hours of science, a fifth was reserved for informal but intense discussions over posters. Scientific sessions of the meeting broadly centered on topics pertaining to membrane structure, mechanics, and physiology.

This special issue is a reflection of the many exciting talks and discussions that were held. Overall, broadly three emerging areas in membrane biology have been discussed in this special issue: membrane and receptor structure, membrane mechanics, and membrane physiology. This meeting has been an important catalyst to foster new and sustainable international networks in membrane biology, especially among early career investigators. This special issue has been subdivided into three sub parts and highlights current research in experimental and computational membrane biology.

We start our special issue with an overall perspective on the role of lipids and membrane assemblies on prebiotic life. Rajamani and co-workers (Sarkar et al. 2020a) have comprehensively discussed current work on prebiotic lipids and membranes in terms of their probable origin, composition, function as well as evolution. They outline how prebiotic membranes could have evolved in response to selection pressures on the early Earth based on their respective physicochemical and self-assembly properties. The diversity of lipids reported in cells and cell membranes continues to grow, and it is becoming extremely clear that both specific lipids and their relative abundance play an important role in cell physiology. Kamat and co-workers describe here one of the newest members of biologically important lysophospholipids, lysophosphatidylserines (lyso-PSs) whose metabolic deregulation has been linked to various human pathophysiological conditions (Shanbhag et al. 2020). They discuss how these lipids were detected in biological systems, their biodistribution and biological pathways directly regulated 
by lyso-PSs in mammals. Dadhich and Kapoor generalize these concepts in the context of different pathogenic lipids in infectious diseases, in particular in exploring virulent lipid-host interactome (Dadhich and Kapoor 2020). They consider recent work on several microorganisms including STEC, MTb, HIV, and sars-cov-2 and substantiate the claim that lipids act as critical determinants of bacterial and viral pathogenesis by altering the host cell membrane structure and functions. Pattnaik and Chakraborty reiterate that not just specific lipids but lipid assemblies are critical determinants of viral pathogenesis (Pattnaik and Chakraborty 2020). In particular, they describe the lipid-stalk model of membrane fusion and show how inhibitors of fusion and membrane topology changes can limit viral entry. These peptide and small molecule-based entry inhibitors could act as broad-spectrum antiviral agents that have added significance in the light of the COVID-19 pandemic to shift our attention toward broad-spectrum antiviral agents from the so-called 'one bug-one drug' approach.

Going up the scale, Srivastava and co-workers have described design principles underlying membrane organization and how they modulate protein localization (Bodosa et al. 2020). Several membrane proteins have been shown to preferentially localize into specific membrane phases and their review highlights the importance of this preferential localization in terms of underlying functions. They emphasize the need to understand the molecular designlevel features that can help us "engineer" nano/microdomain-specific peptides and proteins with varying functional forms. Similarly, Pawar and Sengupta have characterized the nanodomain organization in the vicinity of ErbB2 growth factor receptor transmembrane dimer and reported how cholesterol can alter the local membrane phase (Pawar and Sengupta 2021). They decipher a complex interplay of specific and non-specific effects in the transmembrane domain dimerization using advanced sampling molecular dynamics approaches. Membrane partitioning and protein localization for surface-bound proteins are still emerging. In their work, Srinivasan, Srivastava, and co-workers identify a possible membrane-targeting sequence, a predicted amphipathic helix, at the C-terminus of the protein SopA that belongs to the ParA superfamily of proteins (Mishra et al. 2021). Using a combined experimental and computational approach, they show that the predicted amphipathic helical motif of SopA has weak affinity for membranes and it can associate with bacterial membranes, as detected in the membrane fractions of bacterial lysates.

Membrane receptors such as $G$ protein-coupled receptors (GPCRs) are the main hubs in several signaling pathways originating at the membrane. A deeper analysis of the structural dynamics of these receptors is not only necessary for therapeutic design, but also for deciphering the interplay between the membrane and the receptors. Joshi and co-workers use molecular modeling approaches to analyze insect GPCRs and design targeted ligands with potential insecticide properties (Gujar et al. 2021). In their work, they focus on the orthosteric ligand binding site situated at the lumen of the receptor. Ligand binding at allosteric sites is an emerging area in GPCR biology that promises to open new avenues in understanding signaling regulation as well as therapeutic design. Karnik and co-workers have critically analyzed the current understanding of allosteric modulators and examined current GPCR experimentally resolved structures to compile possible allosteric sites and mechanisms (Singh and Karnik 2021). Interestingly, GPCRs have now been shown to signal not just from the cell surface, but also from the intracellular locations. Puthenveedu and co-workers describe here emerging concepts in compartmentalized GPCR signaling from intracellular membranes (Crilly and Puthenveedu 2020). They conceptualize the "spatial bias" in GPCR signaling and present the evidence for GPCR signaling through $\mathrm{G}$ protein effectors from intracellular membranes and the ways in which this signaling differs from canonical plasma membrane signaling with important implications for physiology and pharmacology. Receptor-lipid interactions are a cornerstone in receptor biology dictating receptor stability and structural dynamics. Chattopadhyay and co-workers report on the physico-chemical mechanisms underlying the interaction of sterols with GPCRs (Sarkar et al. 2020b). They have considered two immediate biosynthetic precursors of cholesterol, 7-dehydrocholesterol (7-DHC) and desmosterol, and showed that GPCR activity is sterol dependent. Overall, their results demonstrate the relevance of optimal membrane cholesterol in maintaining the activity of specific GPCRs, such as the serotonin ${ }_{1 \mathrm{~A}}$ receptor.

In general, protein assemblies in membranes have a critical crosstalk with the membrane. In her work as a part of this special issue, Bondar presents a few interesting concepts in this regard, with emphasis on the intramembrane proteases (Bondar 2020). The protease, GlpG, has been suggested to exhibit lipid-coupled dynamics that modulates the binding of its transmembrane substrate. The work highlights the direct binding of phosphatidylglycerol lipids to GlpG and provides insights into protein-lipid interactions in proteases. Another class of protein assemblies that have been highlighted in this special issue are the pore-forming proteins/toxins (PFPs/PFTs) that are expressed by bacteria to primarily cause infections in the host cell. In general, they share very little sequence similarity with each other, and the common mechanisms remain unclear. The review by Chattopadhyay and co-workers provides examples of diverse groups of membrane-damaging PFPs/PFTs in an attempt to uncover common guiding principles that govern the sequence-to-structure paradigm (Mondal et al. 2020). Cheerla and Ayappa take up one example, cholesteroldependent cytolysins (CDCs) which are a class of PFP/PFT 
that requires membrane cholesterol to perturb the membrane via pore formation (Cheerla and Ayappa 2020). They use allatom molecular dynamics simulations and provide molecular-level insights into the inhomogeneity and perturbation induced in membranes by CDCs. They suggest that the mechanisms represent trends across PFTs in the broad CDC subclass of proteins. Another important family of membrane protein assemblies are membrane transporters that allow the transport of cargo such as ions via a "pore-like" channel in the membrane. Gupta and co-workers have analyzed copper transporters and have discussed common structural and unique regulatory properties between members of the subfamily (Mandal et al. 2020). They have attempted to understand the diversity and similarity of regulation and functioning of the members. Due to the close interplay between membrane composition and the embedded membrane protein assemblies, pathogenic bacteria with unique membrane compositions display unique conformational dynamics of their membrane protein assemblies. In their review, Bendre et al. discuss recent progress in structural characterization of several large membrane protein assemblies from Mycobacterium tuberculosis (Mtb), employing cryo-Electron microscopy technique (Bendre et al. 2021). The paper discusses on the potentials of the structural information on drug discovery.

Understanding the principles of cell mechanics and how cells generate, maintain, and deform the membrane structures is critical to analyze overall cell shape and organization. One such mechanistic process is membrane tubulation that is mediated by forces applied on the membrane either due to motor proteins, by polymerization of the cytoskeleton, or due to the interactions between membrane proteins binding onto the membrane. Rangamani and co-workers (Mahapatra et al. 2021) present a comprehensive review of experimental observations of tubule formation and provide context from the framework of continuum modeling. Bose and Chakrabarti have focused on one aspect of membrane-cytoskeleton interactions, namely with spectrin (Bose and Chakrabarti 2020). They hypothesize that the chaperone activity is derived from the surface-exposed hydrophobic patches present in individual "spectrin-repeat" domains and show a competition between the membrane phospholipid binding functionality and chaperone activity of spectrin. Balasubramanian and co-workers focus on another aspect of membrane mechanics and the emerging roles of caveolin as a mechanosensor (Buwa et al. 2020). They describe the mechanosensitive nature of caveolae and focus on the role of pY14Cav1 in regulating cellular mechanotransduction. However, our understanding of membrane mechanics is still limited and general principles are yet to be understood. One of the main reasons limiting our understanding of cell mechanics is the absence of appropriate molecular tools. New synthetic tools, such as those using the principles of
DNA nanotechnology, have helped researchers probe, program, and re-program membrane remodeling and associated phenomena. Bhatia and co-workers have put together a comprehensive review of DNA nanodevices that can be used to probe membrane remodeling and the trafficking machinery (Rajwar et al. 2020).

Overall, this meeting has been an important catalyst to foster new and sustainable international networks in membrane biology, especially among early career investigators. To facilitate the dissemination of the science that was discussed at the meeting and the enthusiasm shared by participants, we have put together this special issue of the Journal of Membrane Biology titled 'Molecular Interplay at the Membrane' that highlights current research in experimental and computational membrane biology.

Acknowledgements We thank the authors of the special issue and Prof. A. Ladokhin for the support. We thank the organizations (Company of Biologists), scientific journals/societies (Royal society of Chemistry, American Chemical Society, FEBS, and DAAD), and corporate sponsors (Gatan, STP Labtech, Biotech desk/Genscript, and Avanti/Sigma) for supporting the meeting.

\section{References}

Bendre AD, Peters PJ, Kumar J (2021) Recent insights into the structure and function of mycobacterial membrane proteins facilitated by Cryo-EM. J Membrane Biol. https://doi.org/10.1007/ s00232-021-00179-w

Bodosa J, Iyer SS, Srivastava A (2020) Preferential protein partitioning in biological membrane with coexisting liquid ordered and liquid disordered phase behavior: underlying design principles. J Membr Biol. https://doi.org/10.1007/s00232-020-00150-1

Bondar AN (2020) Phosphatidylglyerol lipid binding at the active site of an intramembrane protease. J Membr Biol 253(6):563-576

Bose D, Chakrabarti A (2020) Multiple functions of spectrin: convergent effects. J Membr Biol 253(6):499-508

Buwa N, Mazumdar D, Balasubramanian N (2020) Caveolin1 tyrosine-14 phosphorylation: role in cellular responsiveness to mechanical cues. J Membr Biol 253(6):509-534

Cheerla R, Ayappa KG (2020) Molecular dynamics study of lipid and cholesterol reorganization due to membrane binding and pore formation by Listeriolysin O. J Membr Biol 253(6):535-550

Crilly SE, Puthenveedu MA (2020) Compartmentalized GPCR signaling from intracellular membranes. J Membr Biol. https://doi.org/ 10.1007/s00232-020-00158-7

Dadhich R, Kapoor S (2020) Various facets of pathogenic lipids in infectious diseases: exploring virulent lipid-host interactome and their druggability. J Membr Biol 253(5):399-423

Gujar N, Nikte SV, Joshi RS, Joshi M (2021) Molecular characterization of the $\beta$ 2-like octopamine receptor of Helicoverpa armigera. J Membrane Biol. https://doi.org/10.1007/s00232-021-00172-3

Mahapatra A, Uysalel C, Rangamani P (2021) The mechanics and thermodynamics of tubule formation in biological membranes. $\mathrm{J}$ Membr Biol. https://doi.org/10.1007/s00232-020-00164-9

Mandal T, Kar S, Maji S, Sen S, Gupta A (2020) Structural and functional diversity among the members of CTR, the membrane 
copper transporter family. J Membr Biol 253:459-468. https:// doi.org/10.1007/s00232-020-00139-w

Mishra D, Pahujani S, Mitra N, Srivastava A, Srinivasan R (2021) Identification of a potential membrane-targeting sequence in the C-terminus of the F plasmid segregation protein SopA. J Membr Biol. https://doi.org/10.1007/s00232-020-00157-8

Mondal AK, Verma P, Lata K, Singh M, Chatterjee S, Chattopadhyay K (2020) Sequence diversity in the pore-forming motifs of the membrane-damaging protein toxins. J Membr Biol 253(5):469-478

Pattnaik GP, Chakraborty H (2020) Entry inhibitors: efficient means to block viral infection. J Membr Biol 253(5):425-444

Pawar AB, Sengupta D (2021) Role of cholesterol in transmembrane dimerization of the ErbB2 growth factor receptor. J Membr Biol. https://doi.org/10.1007/s00232-021-00168-z

Rajwar A, Morya V, Kharbanda S, Bhatia D (2020) DNA nanodevices to probe and program membrane organization, dynamics, and applications. J Membr Biol. https://doi.org/10.1007/ s00232-020-00154-x
Sarkar S, Das S, Dagar S, Joshi MP, Mungi CV, Sawant AA, Patki GM, Rajamani S (2020a) Prebiological membranes and their role in the emergence of early cellular life. J Membr Biol 253(6):589-608

Sarkar P, Jafurulla M, Bhowmick S, Chattopadhyay A (2020b) Structural stringency and optimal nature of cholesterol requirement in the function of the serotonin 1A receptor. J Membr Biol 253(5):445-457

Shanbhag K, Mhetre A, Khandelwal N, Kamat SS (2020) The lysophosphatidylserines-an emerging class of signalling lysophospholipids. J Membr Biol 253(5):381-397

Singh KD, Karnik SS (2021) Current trends in GPCR allostery. J Membr Biol. https://doi.org/10.1007/s00232-020-00167-6

Publisher's Note Springer Nature remains neutral with regard to jurisdictional claims in published maps and institutional affiliations. 